Journal of Advanced College of Engineering and Management, Vol. 4, 2018

\title{
EFFECT OF SYSTEM PARAMETERS ON NATURAL FREQUENCY OF THE PELTON TURBINE
}

\author{
Laxman Motra $^{1}$, Sanjeev Karki ${ }^{2}$ \\ ${ }^{1}$ Department of Mechanical Engineering, Pulchowk Campus, Institute of Engineering, Tribhuvan University, \\ Nepal \\ ${ }^{2}$ Department of Applied Science, Advanced College of Engineering and Management, Institute of Engineering, \\ Tribhuvan University, Nepal
}

Email Address: laxman.motra@pcampus.edu.np, sanjeev.karki@acem.edu.np

\begin{abstract}
The effect of diameter, length and rotational speed of shaft, and mass of runner-buckets assembly on the natural frequency of the Pelton turbine unit was analyzed. Effect of the decisive parameters on the natural frequency of the unit showed that it was directly proportional to the diameter of the shaft and inversely proportional to the length of the shaft and mass of the runner-buckets assembly. For the continuous system model, when the rotational speed of the shaft increased, the natural frequency for the forward whirl also increased but the natural frequency decreased for the backward whirl.
\end{abstract}

Keywords: Natural frequency, resonance, discrete, continuous, effect

\section{Introduction}

Rotors and rotating parts are key components with vital role in various engineering applications like pump, compressors, turbines, generators, fans, marine drives and many more. Parts of rotor-blade systems are subjected to highly hostile working conditions. Thus, the design and manufacturing challenge is concerned with the improvement in performance, life span and weight reduction without loss of reliability. The possible forces responsible for the vibration increase in a hydro turbine may be mechanical, hydraulic or electrical. These forces may be mechanical excitations, centrifugal forces due to imbalance of the rotating mass i.e. runner, shaft, and generator rotor, elastic force of the shaft due to incorrect shaft alignment, frictional forces, oil-film instability in bearing, hydraulic excitations, flow through waterways; non-uniform velocity distributions in various waterways of the turbine cause hydraulic unbalance and pressure fluctuations in the penstock and electrical excitations etc [1]. Among the different hydraulic turbines, Pelton turbine is the widely used turbine worldwide; it is appropriate in large, medium and small hydropower plants with large head and low discharge [2]. With the increasing capacity and size of the turbines, the vibration cause by the turbine structure and rotating system strongly influences the safety of the system and may lead to the failure of the system [3]. When an excitation is given and the system is left to vibrate on its own, the frequency at which the unit will oscillate is its natural frequency. If the natural frequency of the rotor-bearing system coincides with the frequency of the periodic excitation generated by the rotor, then the corresponding speed is called the critical speed of the system. When the frequency of the external excitation coincides with the natural frequency of the system, then resonance occurs, which results in the maximum amplitudes of vibration. The frequency location of the critical speed is defined as the frequency of the peak vibration response. Resonance occurs leading to the dangerously large oscillations and may cause excessive deflection and failure. Sometimes the failure of structures like buildings, bridges, turbines, and airplane wings etc. are associated with the occurrence of resonance [4]. Thus, the calculation of the natural frequencies of any system is one of the important parts of vibration study and analysis. 
ANSYS finite element software was used to model the main shaft system in the hydro-turbine generating unit [5]. The key idea of FEM is to transform the infinite degrees of freedom problem into the finite DOF's problem, finally, solving it. They performed modal analysis to calculate the critical speed of rotation. Their research work concluded that, as the operating speed of the shaft increased, the natural frequency of the positive whirl also increased while that of negative whirl decreased.

The detuning procedure was recommended to prevent resonances of Pelton runner water turbines[6]. They have shown that a mathematical model of the runner was obtained from a vibration measurement executed on the finished runner. The masses of the buckets were reduced by grinding on the proper locations on the backside of the buckets very specifically within the allowable mass unbalance range. By detuning of the buckets, the natural frequency of the system was increased without much affecting the stiffness of the buckets and the structural integrity of the runner. Three turbines with the $29000 \mathrm{~kg}$ of Pelton runners and $380 \mathrm{~kg}$ buckets, generating $423 \mathrm{MW}$ at rated 428.57 rpm power each, were detuned by reducing the mass of buckets in the range of some kilograms (not more than $2 \mathrm{~kg}$ ) per bucket (some percentage of the bucket's mass), as a result of which the natural frequency was increased from $745.9 \mathrm{~Hz}$ to $764.3 \mathrm{~Hz}$.

The effect of shaft length and bearing arrangement, effect of bearings stiffness and effect of residual mass unbalance on the blade displacement amplitudes for their $1 \mathrm{~kW}$ radial inflow turbine was investigated by ANSYS Parametric Design Language (APDL) [7]. The displacement amplitude reduces with decreasing shaft length and bearing span, amplitude increases with increasing unbalance mass and displacement amplitudes increases in nonlinear manner with the reduction in bearing stiffness. The relation of the displacement amplitudes with the critical speed represented that critical speed increases with the increment of the shaft diameter and decreases with the increment in the length of the shaft and decreases with the increment in the stiffness of the bearings.

\section{Governing Equations for undamped Free Vibration}

Natural frequency of the Pelton turbine has been evaluated by both discrete and continuous system models. Comparison of one discrete system model which gave close result with the continuous system model has presented.

\subsection{Equation of Motion by Rayleigh's Energy Method: Effective Mass Models}

The mass of the continuous system is distributed throughout the system and have infinite number of degrees of freedom. Analytical solutions to the equations of continuous system are often difficult to obtain. Thus, approximate discrete systems of the continuous systems are often used to approximate the vibration properties. Although treatment of a system as continuous gives exact results, most of the practical systems are treated as discrete system. More accurate results are obtained by increasing the number of masses and increasing the number of degrees of freedom when continuous system is approximated by discrete system with finite number of degrees of freedom [3].

The principle of conservation of energy (kinetic and strain energy) is used to evaluate the effective mass of the system. This method is employed for the lower degrees of freedom systems, mostly single degree of freedom systems. If the mass of the elastic member or spring is much smaller than the mass of the disk, its kinetic energy is negligible with respect to the disk, then inertial effects of member has negligible effect on the motion of the system and system is modeled as one degree of freedom system. If the mass of the elastic member is comparable to the mass of the disk, considering one degrees of freedom model may not give good approximation. The elastic member should be modeled as a continuous system. If the mass of the member is much smaller than the mass of the disk, but not 
negligible, a reasonable one degree of freedom approximation can be made by approximating the inertial effects of the elastic members i.e. effective mass of the elastic member.

\section{Natural Frequency with Simple Supports at Ends}

A mass $M_{D}$ of the rotor disk (runner-buckets assembly) is mounted at the axial center of the shaft of stiffness $K_{s}$. The mass of the shaft is $m_{s}$ and length $L$. For the simply supported uniform shaft, considering the effective mass of the shaft also, the undamped natural frequency of the system is expressed as [3],

$$
\omega_{n}=\sqrt{\frac{K_{s}}{\left(M_{D}+0.4857 m_{s}\right)}}=\sqrt{\frac{48 E I}{\left(M_{D}+0.4857 m_{s}\right) L^{3}}}
$$

Equation 1

\subsection{Equation of Motion by Continuous System Model}

The governing equations for the vibration of Pelton turbine unit were formulated for the continuous system model by using Lagrange's equation. The model was developed by considering flexible continuous and distributed mass shaft and lumped mass of disk concentrated at the middle of the shaft which was simply supported at ends.

The natural frequencies for continuous system model are given as [8],

$\Omega_{1 c r}=\omega_{1 n}=\sqrt{\frac{K}{(m+a)}}$

$\Omega_{2 c r}=\omega_{2 n}=\sqrt{\frac{K}{(m-a)}}$ Equation 2

Where, $\Omega_{1 c r}\left(\omega_{1 n}\right)$ and $\Omega_{2 c r}\left(\omega_{2 n}\right)$ are the critical speeds or natural frequencies of the system for backward whirl and forward whirl respectively.

$$
\begin{aligned}
& m=M_{D}+\frac{\rho A L}{2}+\frac{\rho I \pi^{2}}{2 L} \\
& a=\frac{\rho I \pi^{2}}{L} \\
& K=\frac{\pi^{4} E I}{2 L^{3}}
\end{aligned}
$$

Equation 3

Equation 4

Equation 5

Where, $\rho$ is the mass per unit volume of shaft material, $A$ is the cross-sectional area of shaft and it was assumed to be constant, $L$ is the length of the shaft, $I$ is the area moment of inertia of the shaft crosssection about the neutral axis and it was also supposed to be constant (i.e. for symmetrical shaft, $I_{x x}=$ $I_{z z}=I=\pi D^{4} / 64, D$ is diameter of shaft, $E$ is the Young's modulus of elasticity of the shaft material).

Forward whirling indicates that the lateral vibration follows the direction of the shaft rotation, and the backward whirl represents the vibration that moves in the opposite direction of the shaft movement.

\section{Results and Analysis}

As the Rayleigh's energy method (REM) for simply supported shaft at ends has given the close result of the critical frequency to the continuous system model, the effect of decisive parameters on natural frequency has been analyzed for these models only. The trend of variation of the natural frequency of the continuous system for the forward whirl (FW) and backward whirl (BW) were similar and very 
close to each other while varying the diameter, length of the shaft and mass of the runner-buckets assembly. So, for the clarity during the analysis phase, only forward whirl values were taken for the variation analysis [8].

\subsection{Variation of Natural Frequency with the Diameter of Shaft}

One of the main components of the Pelton turbine unit is shaft, which highly affects the dynamic response of the unit. The influence of the diameter of shaft on the critical frequency was assessed according to Equation 1 and Equation 2 for continuous system and Rayleigh's energy method respectively provided that the other parameters remained constant. Fig 1 presents the variation of critical frequency with the diameter of the shaft.

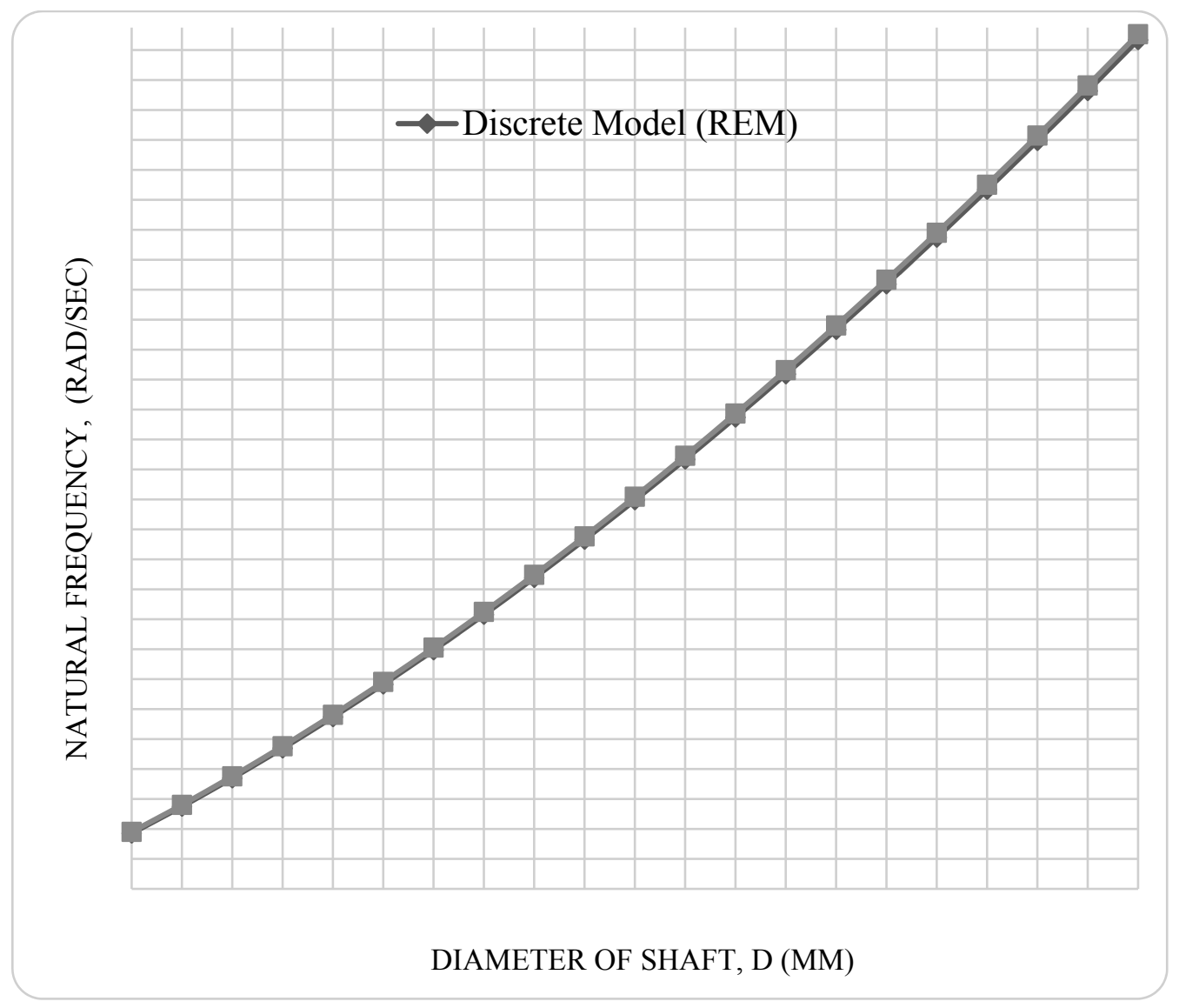

Fig 1: Variation of natural frequency with shaft diameter

Fig 1 illustrates that with the increase in the diameter of the shaft, the natural frequency of the unit also increases for both cases. As $I$ is directly proportional to the diameter of the shaft, it directly affects the natural frequency of the system. This is because with the increment in the diameter of the shaft, as per Equation 1 and Equation 5, the stiffness of the shaft also increases which result in the increment of the natural frequency. If the shaft was designed with higher value of factor of safety, which would result in the large size of the shaft, the natural frequency of the unit would be increased and be much higher than the operating speed of the unit.

\subsection{Variation of Natural Frequency with the Mass of Runner-Buckets}

Another important parameter affecting the natural frequency of the unit is mass of the runner-buckets assembly. The variations of mass of runner-buckets assembly $M_{D}$ directly affect the value of $m$ as per Equation 3 and $m$ is inversely related to the natural frequency of the system according to Equation 2 . The variation of the natural frequency with the mass of runner-buckets assembly was examined 
according to Equation 1 and Equation 2 for the discrete Rayleigh's energy method and continuous system respectively. Fig 2 shows the variation of natural frequency with the mass of runner-buckets assembly keeping other parameters constant.

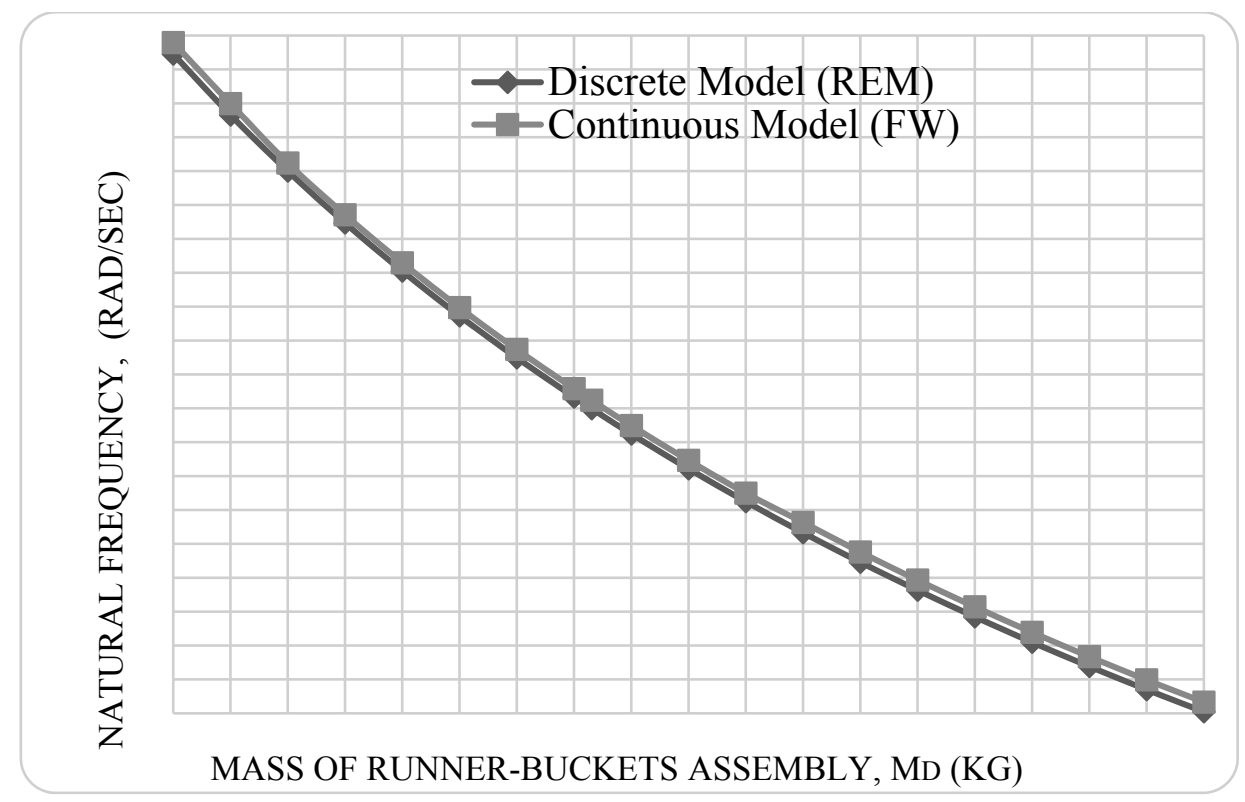

Fig 2: Variation of natural frequency with mass of runner-buckets assembly

The relationship between the natural frequency of the unit and the mass of the runner-buckets assembly show that natural frequency reduces with the increase in mass of the runner-buckets assembly which is clearly represented by Fig 2 . Due to this relationship, the detuning of the buckets is possible to increase the natural frequency of the system without seriously affecting the stiffness of the buckets and the structural integrity of the runner and it is done by reducing the mass of each buckets.

\subsection{Variation of Natural Frequency with the Length of Shaft}

Length of shaft is another parameter that also affects the dynamic response of the unit. According to Equation 1 and Equation 2, values of natural frequencies were calculated for various length of shaft for discrete Rayleigh's energy method and continuous system models.

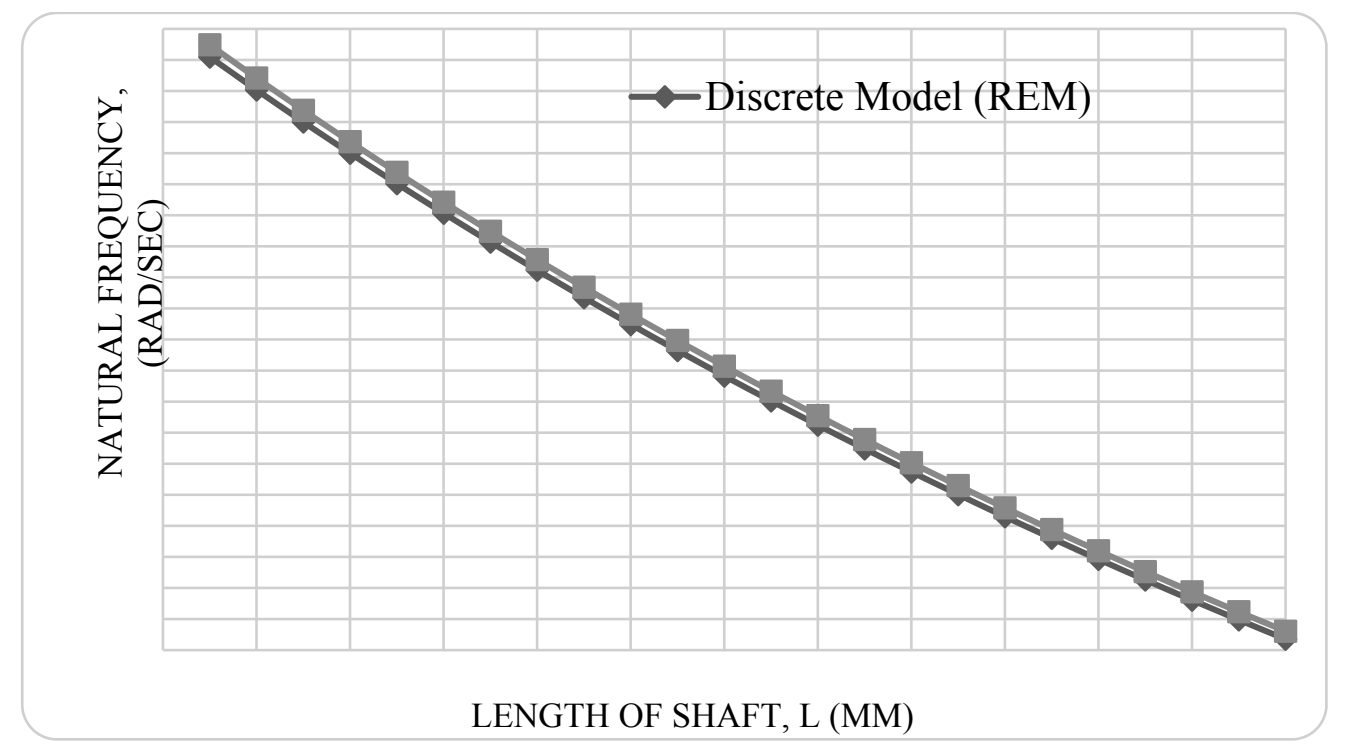

Fig 3 : Variation of natural frequency with length of shaft. 
Fig 3 depicts the variation of the natural frequency with the length of shaft. It shows that with the increase in the length of the shaft, the natural frequency of the system decreases. According to Equation 1 and Equation 5, stiffness of the shaft is inversely proportional to the cubic power of the length $\left(L^{3}\right)$, which resulted in such output relationship. By decreasing the length of shaft i.e. bearing to bearing distance, the natural frequency of the unit can be increased but there is limitation for reducing the size of runner-buckets assembly casing due to the striking of water jets, splashing water management system and the placement of nozzles. But, the length of the shaft and bearings span should be investigated by considering the physical limitation in installation.

\subsection{Variation of Natural Frequency with the Rotational Speed of Shaft}

Natural frequencies for the continuous system model were assessed according to the variation of speed of the rotation of the shaft for backward whirl (BW) and forward whirl (FW) as per Equation 2. When the rotational speed of the shaft was varied from $500 \mathrm{rpm}$ to $30000 \mathrm{rpm}$, little variation was found in the natural frequency of the shaft. It varied from $542.732 \mathrm{rad} / \mathrm{sec}$ to $543.7 \mathrm{rad} / \mathrm{sec}$ for forward whirl and $542.7 \mathrm{rad} / \mathrm{sec}$ to $541.734 \mathrm{rad} / \mathrm{sec}$ for backward whirl. The trend of change of the natural frequency for forward whirl is directly proportional to the rotational speed of the shaft, but for the backward whirl it is inversely proportional to the spin speed of the shaft which is represented in Fig 4.

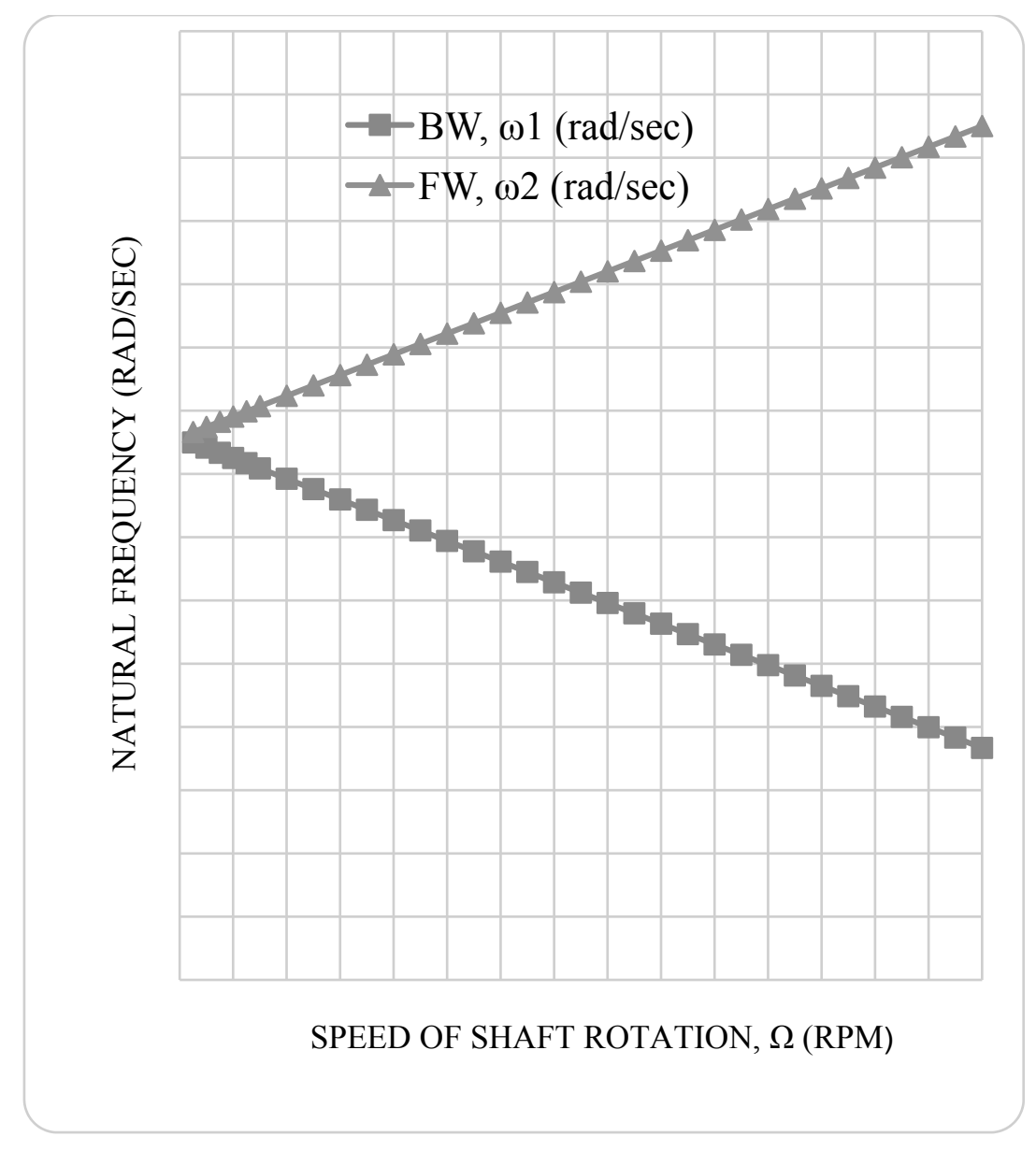

Fig 4: Variation of the natural frequency with rotational speed of shaft 


\section{Conclusions}

The continuous shaft and runner-buckets assembly system can be adequately modeled as a single degree of freedom discrete system with the effective mass of simply supported shaft at ends to determine the critical frequency of the unit with reasonable accuracy. The results depict that natural frequency of the unit is directly proportional to the diameter of the shaft and inversely proportional to the length of the shaft and mass of the runner-buckets assembly. When the rotational speed of the shaft increases, the natural frequency for the forward whirl increases while it decreases for the backward whirl for the continuous system model.

\section{References}

1. Wu, Y., Li, S., Liu, S., Dou, H.S. and Qian, Z., 2013. "Vibration of hydraulic machinery” (pp. 431-476). Berlin: Springer.

2. Harvey, A., 1993. "Micro-Hydro Design Manual: a guide to small-scale water power schemes" (No. 621.24/H341). Intermediate Technology Publications.

3. Thomson, W.T. and Dahleh, M.D., 2005. "Theory of vibration with applications", Fifth Edition. Pearson Education Asia Limited, China, ISBN 978-7-302-12137-4.

4. Rao, S.S, 2013. "Mechanical Vibrations", Fourth Edition. Pearson, India, ISBN 978-81-7758874-3.

5. Bai, B., Zhang, L., Guo, T. and Liu, C., 2012. "Analysis of dynamic characteristics of the main shaft system in a hydro-turbine based on ANSYS”. Procedia Engineering, Vol.31, pp.654-658.

6. Schmied, J., Weiss, T. and Angehrn, R., 2006, September. "Detuning of Pelton runners". In Proceedings of the 7th IFToMM Conference on Rotor dynamics, Vienna, Austria (pp. 2528).

7. Jung, H.C. and Krumdieck, S., 2014. "Rotordynamic modelling and analysis of a radial inflow turbine rotor-bearing system". International journal of precision engineering and manufacturing, 15(11), pp.2285-2290.

8. Motra, L. and Luintel, M. C., 2017. "Free vibration analysis of selected Pelton turbine using dynamic approach”. Proceedings of IOE Graduate Conference, 2017, Vol. 5, pp. 229-236. 